Observation of paral I el vi scosity in a st el I ar at or

\begin{tabular}{|c|c|}
\hline $\begin{array}{l}\text { j our nal or } \\
\text { publ i cat i on } \mathrm{titl} \text { e }\end{array}$ & Physi cal Revi ew Let ter s \\
\hline vol une & Vol . 67 \\
\hline number & No. 1 \\
\hline page $r$ ange & pp. 58-61 \\
\hline year & 1991- 07- 01 \\
\hline URL & ht t p: //hdl . handl e. net /10655/115 \\
\hline
\end{tabular}




\title{
Observation of Parallel Viscosity in a Stellarator
}

\author{
K. Ida, H. Yamada, H. Iguchi, K. Itoh, and CHS Group \\ National Institute for Fusion Science, Nagoya, 464-01, Japan \\ (Received 26 December 1990)
}

\begin{abstract}
Damping of the plasma toroidal velocity owing to parallel viscosity is observed in the Compact Helical System heliotron-torsatron device with tangential neutral-beam injection. The toroidal velocity profile is dominated by perpendicular viscosity when the magnetic-field modulation is weak near the axis. However, parallel viscosity is found to be dominant when the modulation is strong enough, and to increase in proportion to the square of the modulation of the magnetic field. The absolute values of the viscosity agree with the neoclassical prediction within a factor of 3 .

PACS numbers: $51.20 .+\mathrm{d}, 52.30 .-\mathrm{q}, 52.55 . \mathrm{Hc}$
\end{abstract}

In a toroidal plasma, poloidal and toroidal rotation velocities are determined through the balance between the input torque and the damping force in steady state. In tokamak $\boldsymbol{H}$-mode plasmas, ${ }^{1}$ significant poloidal rotation has been observed. ${ }^{2,3}$ In a recent theoretical model, the poloidal rotation is assumed to be driven by the poloidal torque associated with the ion-orbit loss ${ }^{4,5}$ or imposed radial current, ${ }^{6,7}$ and is damped by the neoclassical parallel viscosity. However, the poloidal viscosity has not been measured precisely enough for a comparison with neoclassical theory, ${ }^{8,9}$ because of the difficulty in estimating the poloidal torque. On the other hand, toroidal rotation is mainly driven by the torque given by the neutral beam in plasmas with tangential neutralbeam injection (NBI). There have been many observations of toroidal rotation in tokamaks, ${ }^{10-13}$ but the damping is mainly due to an anomalous perpendicular viscosity (radial diffusion of momentum). Because the magnetic-field ripple in the toroidal direction is so small, the effect of neoclassical parallel viscosity on toroidal rotation is easily masked by the anomalous perpendicular viscosity. In helical devices, the helical ripple is large enough to affect toroidal rotation. The poloidal rotation has been measured on Heliotron-E (Ref. 14) and Wendelstein VII-A (Ref. 15); however, toroidal rotation and discussions on the damping mechanism have not been reported. A preliminary report has been made on the measurements of toroidal and poloidal velocities in the Compact Helical System with tangential neutral-beam injection, and on the viscosity. ${ }^{16}$ In this article, we investigate the toroidal rotation velocity over a wide range of density and magnetic-field ripple, and discuss a comparison of the measurements with neoclassical theory.

The Compact Helical System ${ }^{17}$ (CHS) is a heliotrontorsatron device (poloidal period number $l=2$, and toroidal period number $M=8$ ) with a major radius $(R)$ of $1 \mathrm{~m}$, an average minor radius $(a)$ of $20 \mathrm{~cm}$, and a rotational transform at the plasma edge $\chi_{a} \quad\left(=R B_{\theta} / a B_{\varphi}\right.$, where $B_{\varphi}$ and $B_{\theta}$ are the toroidal and poloidal magnetic fields, respectively) of $\sim 1$. In CHS, the magnetic-field ripple at the plasma center can be changed by shifting the magnetic axis. The major radius of the vacuum magnetic axis $\left(R_{\mathrm{ax}}\right)$, which is calculated in the vacuum magnetic-field configuration, is changed from 89.9 to $101.6 \mathrm{~cm}$ in our experiments. The central helical ripple $\varepsilon_{h}^{m, l}(0)$ is negligible when the major radius is set at 90-95 cm; however, it increases sharply for $R_{\mathrm{ax}}>95 \mathrm{~cm}$ and reaches $8 \%$ at $R_{\text {ax }}=101.6 \mathrm{~cm}$.

Plasma is produced initially by electron cyclotron heating $(\mathrm{ECH})$ with hydrogen gas and sustained with tangential NBI (injection energy of $32.5 \mathrm{keV}$ and absorbed power of $0.5 \mathrm{MW}$ in the direction parallel to the helical current). The line-averaged density reaches around $2 \times 10^{13} \mathrm{~cm}^{-3}$ after NBI. Figure 1 shows the change of profiles of toroidal and poloidal rotation velocities, electron and ion temperatures, electron density, and modulation of magnetic-field strength $\gamma$ as a result of the major-radius scan, which is controlled by the vertical field strength. Here modulation of the magnetic-field strength $\gamma$ is defined as $\gamma^{2}=\left\langle(\partial B / \partial s)^{2}\right\rangle / B^{2}$, where $s$ is the length along the magnetic-field line and \langle\rangle is a fluxsurface-average operator. Profiles of ion temperature and the poloidal and toroidal rotation velocities are measured with charge-exchange spectroscopy using a 1-m visible spectrometer. The spectrometer has two 38channel poloidal and one 38-channel toroidal opticalfiber inputs and provides 128 spectra simultaneously with a two-dimensional charge-coupled-device detector. ${ }^{18,19}$ The charge-exchange spectra at $5290.5 \AA$ for the $n=8$ to $n=7$ transition in $\mathrm{C}^{5+}$ is used for the measurements. Profiles of electron density and temperature are measured with a Thomson-scattering system. We evaluate $\gamma$ by averaging $(\partial B / \partial s)^{2}$ along the field line for one toroidal turn. The structure of the magnetic field is represented using Fourier components with five toroidal mode numbers and seven poloidal mode numbers. The amplitude for each component is derived from the finite- $\beta$ 3D equilibrium code VMEC, ${ }^{20}$ based on the kinetic data in the experiments. As demonstrated on the iontemperature profile, the plasma axis $\left(R_{p}\right)$ is shifted outward due to the finite- $\beta$ effect and this Shafranov shift is $2-5 \mathrm{~cm}$ depending on the plasma and beam pressure and 

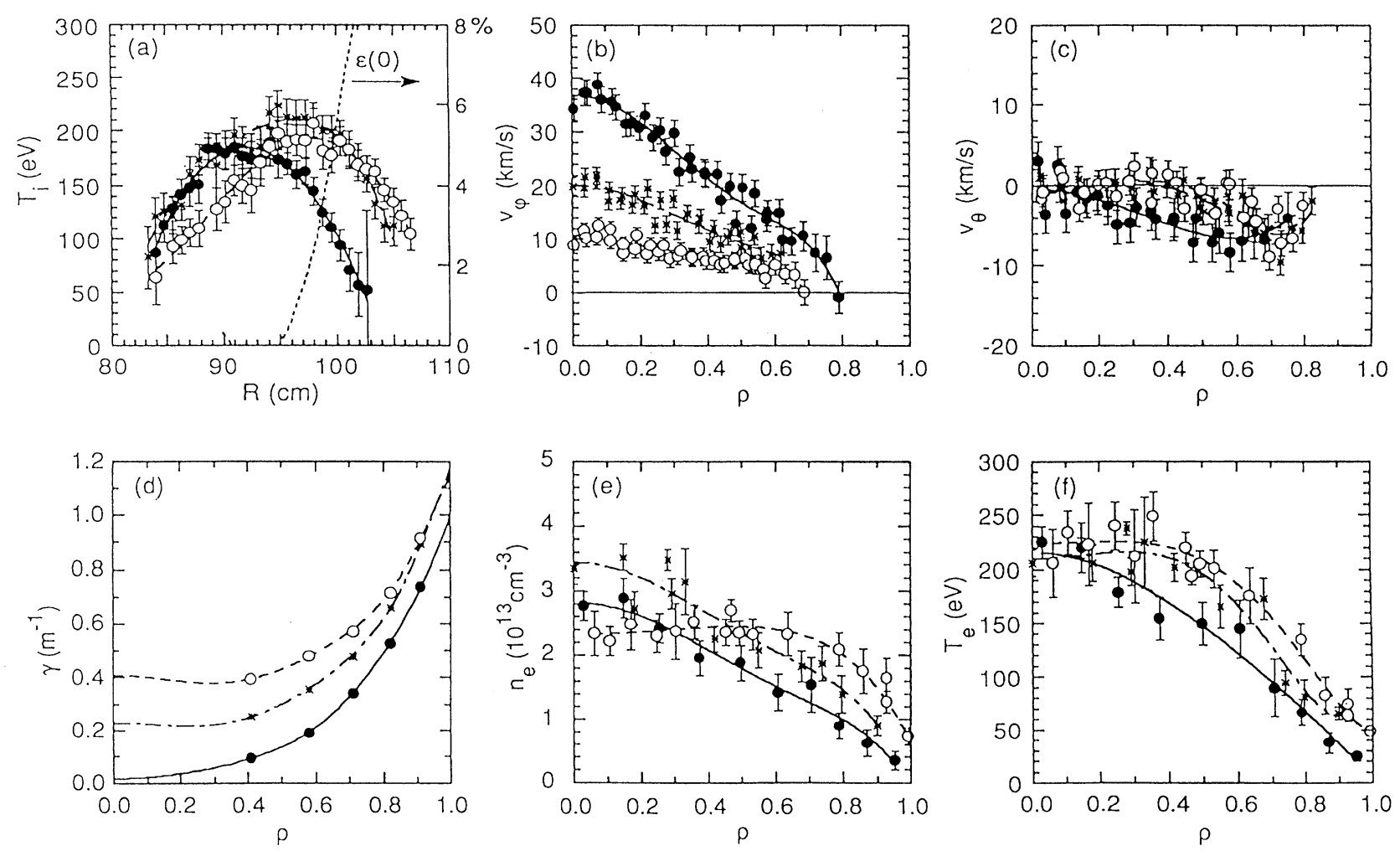

FIG. 1. Radial profiles of (a) ion temperature, (b) toroidal rotation velocity, (c) poloidal rotation velocity, (d) modulation of magnetic-field strength $\gamma$ (where $\left.\gamma^{2}=\left\langle(\partial B / \partial s)^{2}\right\rangle / B^{2}\right)$, (e) electron density, and (f) electron temperature for $R_{\text {ax }}$ of $89.9 \mathrm{~cm}$ (solid circles), $94.9 \mathrm{~cm}$ (asterisks), and $97.4 \mathrm{~cm}$ (open circles), where $\rho$ is the normalized averaged minor radius. The dotted line in (a) represents the fraction of the helical ripple at the plasma center as a function of major radius.

the rotational transform $\chi\left(=R B_{\theta} / a \rho B_{\varphi}\right)$. Details of the measurements of the Shafranov shift in CHS will be reported elsewhere. The torque due to injected fast ions has been estimated for each plasma axis $R_{p}$ using an analytical model, ${ }^{21}$ where the fast-ion birth distribution and the shine-through are calculated. Since the deposition of fast-ion energy declines due to orbit loss and charge-exchange loss, their effects have been included in the model to match the result of the 3D Monte Carlo code HELIOS. 22

The toroidal rotation velocities show significant damping as the axis of the plasma is shifted outward, although the energy confinement time measured with a diamagnetic loop increases from $1.5\left(R_{\mathrm{ax}}=89.9 \mathrm{~cm}\right)$ to $2.5 \mathrm{~ms}$ $\left(R_{\mathrm{ax}}=97.4\right)$. The poloidal rotation velocities also show similar damping behavior in the core region; however, they show no change near the plasma edge $(\rho \sim 0.7)$. The global confinement is improved by shifting the plasma outward, but the particle confinement in the core region decreases, as observed in the electron-density profiles. The noticeable difference in particle transport between the cases with $R_{\text {ax }}=94.9 \mathrm{~cm}$ and with $R_{\text {ax }}=97.4$ $\mathrm{cm}$ seems to be associated with the change of toroidal rotation profiles, but the effect of plasma rotation on parti- cle transport ${ }^{23}$ is out of the scope of this paper and will be discussed elsewhere. We note that the toroidal rotation damping and density profile flattening are also observed when the direction of the magnetic field is reversed.

In the core region where $\rho<0.5$, the plasma rotates almost parallel to the magnetic-field line, as shown in poloidal and toroidal rotation profiles, since the rotational transform in this region is $0.3-0.5$. Several mechanisms can contribute to the damping of the parallel velocity $\left(v_{\|}\right)$, such as transit-time magnetic pumping (TTMP) of the rotating ions due to the modulation of $B$ (parallelviscosity term $\mu_{\|} v_{\|}$), radial diffusion of momentum due to the velocity shear (perpendicular viscosity term $\left.\mu_{\perp} \nabla v_{\|}\right)$, and collision with a neutral particle (chargeexchange momentum loss $n_{0}\left\langle\sigma_{\mathrm{cx}} v\right\rangle_{v_{\|}}$, where $n_{0}$ is the neutral density and $\left\langle\sigma_{\mathrm{cx}} v\right\rangle$ is the charge-exchange rate coefficient). Charge-exchange loss becomes dominant only near the plasma edge, and damping due to the $\mu_{\|} v_{\|}$ and $\mu_{\perp} \nabla v_{\|}$terms becomes important in the region of interest $(\rho<0.6)$.

Since the parallel-viscosity term determines the parallel velocity, not the toroidal velocity, for comparison of the measurements with theory, we derive the velocities 
parallel to magnetic-field lines $\left(v_{\|}\right)$from measured toroidal and poloidal rotation velocities and the rotational transform. The parallel velocity near the plasma center is mainly determined by the perpendicular viscosity for $R_{\mathrm{ax}}=89.9 \mathrm{~cm}$, since the modulation of $B$ is too small to affect plasma rotation near the axis. On the other hand, parallel velocity is damped by the parallel viscosity for $R_{\mathrm{ax}}=97.4 \mathrm{~cm}$, where the modulation of $B$ is $0.4 \mathrm{~m}^{-1}$ at the plasma center, as shown in Fig. 2. To compare the measured parallel velocity with the theoretical estimate, the neoclassical (NC) parallel viscosity is taken from Refs. 24 and 25 . The NC parallel viscosity is proportional to the square of the modulation of $B$ as $\mu_{\|}^{\mathrm{NC}} \propto\left\{(\partial B / \partial s)^{2} / B^{2}\right\} F\left(v_{i}^{*}\right)$, where $v_{i}^{*}$ is the ion normalized collisionality. As estimated in Ref. 25, the parallel viscosity is expressed simply as $\mu_{\|} \mathrm{NC}=C\left\{\left\langle(\partial B / \partial s)^{2}\right\rangle\right)$ $\left.B^{2}\right\}(R / m) v_{\text {th }}$ in the plateau regime, where $C$ is a numeral coefficient. We examine the fitting of measured parallel velocity with $\left(\mu_{\|}, \mu_{\perp}\right)$. The perpendicular viscosity of $\mu_{\perp}=3.5 \mathrm{~m}^{2} / \mathrm{s}$ in addition to the $\mathrm{NC}$ parallel viscosity gives the best fit to the measured parallel velocity for $R_{\text {ax }}=89.9 \mathrm{~cm}$; the latter mechanism affects plasma rotation only when $\rho>0.6$. The central rotation velocity may be explained alternatively by increasing the
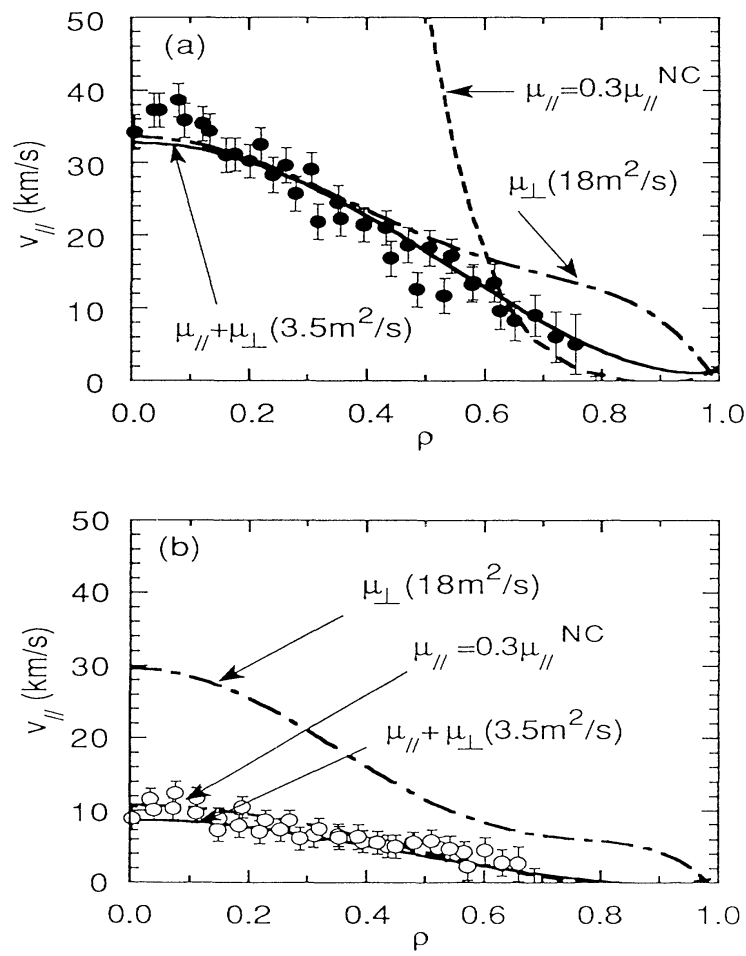

FIG. 2. Radial profiles of the parallel velocity with estimates of velocity by the neoclassical parallel viscosity (dashed line), by radial diffusion of the momentum (dot-dashed lines), and by a combination of the parallel viscosity and radial diffusion (solid line) for major radius of (a) $89.9 \mathrm{~cm}$ and (b) $97.4 \mathrm{~cm}$, where $\mu_{N}{ }^{\mathrm{NC}}$ is parallel viscosity in the plateau regime. values of $\mu_{\perp}$ up to $18 \mathrm{~m}^{2} / \mathrm{s}$ with $\mu_{\|}=0$; however, the radial momentum diffusion alone cannot explain the damping of the parallel velocity at $\rho>0.6$. The explanation using the large perpendicular viscosity alone cannot explain the significant change in the velocity between $R_{\text {ax }}=89.9 \mathrm{~cm}$ and $R_{\text {ax }}=97.4 \mathrm{~cm}$ either. In the case of $R_{\text {ax }}=97.4 \mathrm{~cm}$, the parallel viscosity becomes dominant and the additional perpendicular viscosity changes the estimate of velocity by only $20 \%$. The best fit of the parallel-viscosity profile for $R_{\mathrm{ax}}=97.4 \mathrm{~cm}$ gives $\mu_{\|}=3 \times 10^{3} \mathrm{~s}^{-1}$ which is smaller than the calculation of Shaing by a factor of 3 . The perpendicular viscosity needed to fit the experimental data is comparable to the measured ion thermal diffusivity $\left(\sim 5 \mathrm{~m}^{2} / \mathrm{s}\right)$ and larger than that of the neoclassical prediction $\left(\sim 2 \mathrm{~m}^{2} / \mathrm{s}\right)$ by a factor of 2 .

The parameter dependence of the viscosity is studied by changing the plasma density and the field ripple. Toroidal rotation velocity is measured for $R_{\mathrm{ax}}=92.1 \mathrm{~cm}$ (perpendicular viscosity is dominant) and for $R_{\mathrm{ax}}=94.9$ $\mathrm{cm}$ (parallel viscosity becomes important) in the wide range of electron density from $0.7 \times 10^{13} / \mathrm{cm}^{3}$ to 6 $\times 10^{13} / \mathrm{cm}^{3}$. The perpendicular viscosity is found to decrease roughly proportionally to $1 / n_{e}$ as the global confinement is improved by increasing the electron density. This density dependence indicates the existence of anomalous radial diffusion of the momentum as observed in tokamak plasmas. However, the parallel viscosity does not change for this density scan, confirming the plateau neoclassical prediction.

The observation confirms that the parallel viscosity is proportional to the square of the modulation of the magnetic-field strength. Effective viscosity $\mu_{\mathrm{eff}}$ is defined as $\mu_{\mathrm{eff}}^{-1}=v_{\|} m_{i} n_{e}(0) / f_{\mathrm{NBI}}(0)$, where $f_{\mathrm{NBI}}(0) R$ is the torque from NBI. As shown in Fig. 3, this effective parallel viscosity shows a $\gamma^{-2}$ dependence as predicted by the neoclassical theory in the region where the paral-

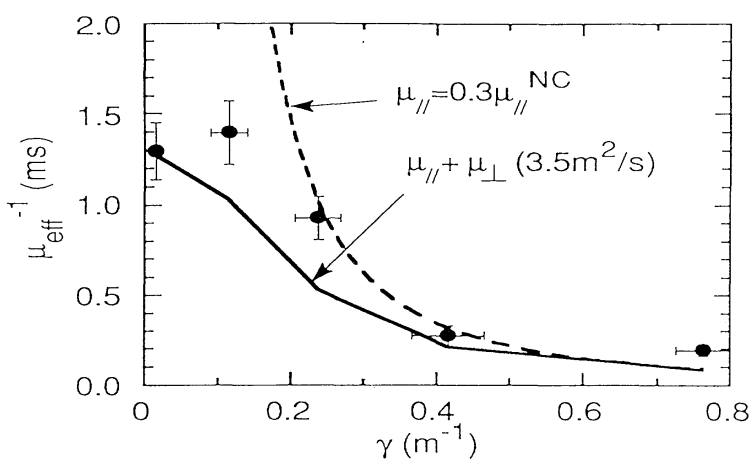

FIG. 3. Inverse central parallel viscosity derived from the measured central parallel velocity, density, and momentum input as a function of the central modulation of magnetic-field strength $\gamma$. The dashed line is an estimate by the neoclassical parallel viscosity and the solid line shows the radial-diffusionof-momentum effect on the measurements. 
lel viscosity becomes dominant, $\gamma>0.2$. When the modulation of $B$ decreases below 0.2 , the parallel velocity becomes small and perpendicular viscosity becomes dominant. Large parallel viscosity and significant chargeexchange loss near the plasma edge become boundary conditions to determine the central parallel velocity in the radial transport of momentum. One of the main errors in evaluating the modulation of $B$ in the measurements is due to the uncertainty of the description of finite- $\beta$ equilibria. The uncertainties of the evaluated modulation of magnetic-field strength are $5 \%-20 \%$ as shown by the error bars in Fig. 3. Finally we estimate the effect of impurities on momentum balance. The impurity concentrations are estimated from VUV spectroscopic measurements. Carbon and oxygen concentrations are $2 \%$ and $1 \%$, respectively, while metallic impurity, dominated by titanium, is $0.1 \%$. These impurities give the $Z_{\text {eff }}$ value of 2 , and increase the estimate of total plasma momentum by $20 \%-30 \%$, compared with the pure hydrogen plasma.

In conclusion, the parallel viscosity is found to increase in proportion to the square of the modulation of magnetic field and show no dependence on plasma density, which are characteristics that agree well with the neoclassical prediction in the plateau regime. Their quantitative agreement is within a factor of 3 . However, when the magnetic-field modulation is weak, the perpendicular viscosity (anomalous radial diffusion of momentum) becomes dominant as in a tokamak plasma.

The authors would like to thank Dr. S. Morita for his help in estimating the impurity content, Dr. S.-I. Itoh, Dr. H. Sanuki, and Dr. Y. Ogawa for elucidating discussions, and the NBI group and the $\mathrm{ECH}$ group for their support of NBI and ECH operation. The authors also thank the computer staff for support in data acquisition. The authors thank Dr. H. C. Howe and Dr. S. P. Hirshman of Oak Ridge National Laboratory for making available their numerical codes, and Dr. K. McCormick of Max-Planck-Institut für Plasmaphysik for correcting the manuscript.

${ }^{1}$ F. Wagner et al., Phys. Rev. Lett. 49, 1408 (1982).

${ }^{2}$ R. J. Groebner, K. H. Burrell, and R. P. Seraydarian, Phys. Rev. Lett. 64, 3015 (1990).

${ }^{3}$ K. Ida, S. Hidekuma, Y. Miura, T. Fujita, M. Mori, K.
Hoshino, N. Suzuki, T. Yamauchi, and JFT-2M Group, Phys. Rev. Lett. 65, 1364 (1990).

${ }^{4}$ K. C. Shaing and E. C. Crume, Jr., Phys. Rev. Lett. 63, 2369 (1989)

${ }^{5}$ S.-I. Itoh and K. Itoh, Phys. Rev. Lett. 60, 2276 (1988).

${ }^{6}$ R. J. Taylor, M. L. Brown, B. D. Fried, H. Grote, J. R. Liberati, G. J. Morales, P. Pribyl, D. Darrow, and M. Ono, Phys. Rev. Lett. 63, 2365 (1989).

${ }^{7}$ R. R. Weynants and R. J. Taylor, Nucl. Fusion 30, 945 (1990).

${ }^{8}$ K. Brau, M. Bitter, R. J. Goldston, D. Manos, K. McGuire, and S. Suckewer, Nucl. Fusion 23, 1643 (1983).

${ }^{9}$ R. D. Benjamin, J. L. Terry, and H. W. Moos, Rev. Sci. Instrum. 57, 2020 (1986).

${ }^{10}$ K. H. Burrell, R. J. Groebner, H. St. John, and R. P. Seraydarian, Nucl. Fusion 28, 3 (1988).

${ }^{11} \mathrm{H}$. Weisen, M. von Hellermann, A. Boileau, L. D. Horton, W. Mandl, and H. P. Summers, Nucl. Fusion 29, 2187 (1989).

${ }^{12}$ A. Kallenbach, H-M. Mayer, G. Fussmann, R. Buchse, O. Gruber, O. Kluber, V. Mertens, O. Vollmer, and H. Zohm, Nucl. Fusion 30, 645 (1990).

${ }^{13}$ K. Ida, S.-I. Itoh, K. Itoh, S. Hidekuma, Y. Miura, N. Suzuki, M. Mori, T. Matsuda, T. Yamauchi, and JFT-2M Group, in Controlled Fusion and Plasma Heating, Amsterdam, 1990 (European Physical Society, Petit-Lancy, Switzerland, 1990), Vol. 14B, Pt. I, p. 267.

${ }^{14}$ K. Kondo et al., Sci. Instrum. 59, 1533 (1988).

${ }^{15} \mathrm{H}$. Wobig, H. Maassberg, H. Renner, WVII-A Team, ECRH Group, and NI Group, in Plasma Physics and Controlled Nuclear Fusion Research, Kyoto, 1986 (IAEA, Vienna, 1987), Vol. II, p. 369.

${ }^{16}$ K. Ida, K. Itoh, S.-I. Itoh, S. Hidekuma, JIPPT-IIU, and CHS Group, in Plasma Physics and Controlled Nuclear Fusion Research, Washington, 1990 (IAEA, Vienna, 1990), IAEA-CN-53/C-3-3.

${ }^{17} \mathrm{O}$. Kaneko et al., in Plasma Physics and Controlled Nuclear Fusion Research (Ref. 16), IAEA-CN-53/C-1-4.

${ }^{18} \mathrm{~K}$. Ida and S. Hidekuma, Rev. Sci. Instrum. 60, 876 (1989).

${ }^{19}$ K. Ida, H. Yamada, H. Iguchi, S. Hidekuma, H. Sanuki, K. Yamazaki, and CHS Group (to be published).

${ }^{20}$ S. P. Hirshman, W. van Rij, and P. Merkel, Comput. Phys. Commun. 43, 143 (1986).

${ }^{21}$ J. A. Rome, J. D. Callen, and J. F. Clarke, Nucl. Fusion 14, 141 (1974).

${ }^{22}$ R. H. Fowler, R. N. Morris, J. A. Rome, and K. Hanatani, Nucl. Fusion 30, 997 (1990).

${ }^{23}$ S.-I. Itoh, J. Phys. Soc. Jpn. 59, 3431 (1990).

${ }^{24}$ T. H. Stix, Phys. Fluids 16, 1260 (1973).

${ }^{25}$ K. C. Shaing and J. D. Callen, Phys. Fluids 26, 1526 (1983). 